\title{
Tempera Teaching Method and the Cultivation of Middle School Students Multiple Literacy Ability
}

\author{
Chen qiduan ${ }^{1, \mathrm{a}}, \mathrm{Xu}$ wanhua ${ }^{2, \mathrm{~b}}$ \\ 1 School of Art and Design, Jimei University, Jimei, Xiamen, Fujian, China \\ 2 School of Art and Design, Jimei University, Jimei, Xiamen, Fujian, China \\ a1140751800@qq.com \\ b*619128746@qq.com
}

\begin{abstract}
Teaching art and design in middle school in the modern era fails to cultivate students' creative thinking ability and aesthetic ability and deviates from the law of students' psychological development. This article aims to sort out the historical reasons and evolution of art education literacy and introduces the related concepts and pedagogy of the Tempera teaching method and multiple literacy reading, understanding and communicating through different techniques, and experiencing diverse experiences. The situational differences in art learning can further cultivate students' thinking and ability to create art. We also use "video analysis" to demonstrate the analysis of the "Basic Art" classroom teaching by the researcher. Through comprehensive textual research and personal teaching experience in art education, the Tempera method should be implemented in all classrooms to improve teaching art to modern middle school students.
\end{abstract}

Keywords: Tempera, Multiple Literacy, chemistry, materials

\section{丹培拉教学法对中学生多元识读能力的培养}

\author{
陈其端 $1, \mathrm{a}$ 许婉华 $2, \mathrm{~b}^{*}$
}

\author{
${ }^{1}$ 集美大学美术与设计学院，集美，厦门，福建，中国 \\ ${ }^{2}$ 集美大学美术与设计学院，集美，厦门，福建，中国 \\ 1140751800@qq.com \\ b*619128746@qq.com
}

\section{摘要}

现今中学美术课堂教学方法, 仍是一味追求纯粹的技法训练。千篇一律对着课本临摹的度过美术课, 还有的地 区学校没开设美术课程。这种指导思想与教学方法, 缺乏对学生创造思维能力与审美能力培养, 背离了学生心 理发展规律, 导致了学生没有正确的美术领域学习观念, 本文因当前时代潮流与中学基本教育及美术新课程所 需, 从多元识读的角度进行对教师美术课程教材教法的研究。首先, 本文对丹培拉教学法与多元识读的相关概 念及教学法进行介绍, 彰显现代美术教育强调透过不同技法进行理解与沟通, 体验多样的美术学习之情境差 异, 进而培养学生思考与美术创作的能力。其次, 本文采用「录影分析」为研究方法, 进行对研究者所授「美 术基础」课堂教学之示例分析。最后, 文本综合研究结果与个人在美术教育的教学经验, 丹培拉教学法以及对 中学生多元识读能力培养等相关内容, 对于提升现代中学生美术课堂教学效果具有重要意义。

关键词: 丹培拉，多元识读，录影分析，美术课程，中学

\section{1.前言}

在传统意义上的美术教学识读对学生教学和认 知模式的培养方式已不再满足当今社会发展的新需 求, 人们需要更新传统的美术教学理念, 改革传统的
美术教学模式。在如今, 许多现代画家仍执着于丹培 拉特殊的创作鬼力, 在这样一个工业信息化的时代, 对速度的追求使艺术家不再一味选择传统方式, 现代 绘画语言的加入, 帮助了丹培拉的艺术表现手法发展 向多样化，十分适合主观意愿的表达与理解美术学习。 本文将丹培拉这一特殊的绘画技法融入美术教育识 
读中, 使现代中学生美术教育识读得到创新与发展。

\section{2.丹培拉教学法及“多元识读”相关阐述}

中学美术教材是课堂教学模式研究的纲本, 故作 为丹培拉教学法对中学生多元识读能力的培养教学 模式革新设计研究重点, 应大力推进丹培拉教学法在 中学美术课程中的开发。本文把多元识读 ${ }^{[1]}$ 能力定义 为: 学习者在已有的认知学习知识和经历, 运用创造

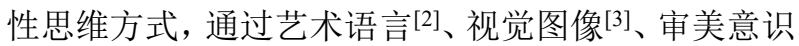
[4]等, 在美术教育与文字认知方式相互融合的过程中, 也以一种新的认知方式开始进入学生的生活和思维 的空间, 即丹培拉教学法认知学习方式。

丹培拉的美术教学课程设置, 与美术专业领域的 审美素养与艺术创作能力密切结合, 充分对中学生进 行有实际操作的、有艺术文化多元识读认知的训练, 中学美术老师要对关于丹培拉课程资源有一个整体 的规划和开发。特别是在全球经济文化一体化现象突 出的背景下, 中学生掌握了一定的人文和社会学科的 知识后, 对于民族艺术传统文化和艺术绘画语言多元 化会有不一样的诠释和理解, 关注中学生真个年龄的 精神追求和审美情趣, 为丹培拉教学法对多元识读的 培养设下夯实基础。引导中学生如何正确地对待艺术 文化以及从各种门类的艺术领域中汲取营养, 丰富自 身的人文社科知识, 更好的利用关于丹培拉教学法在 多元识读的资源是教学的重点。在具体的操作实施过 程中, 在原有的中学美术教学框架结构及美术课标的 要求下, 采用丹培拉绘画语言及技法应用其中, 凸显 艺术文化专业化、丹培拉材料特色, 能动地将其融合 于教材, 为基于丹培拉教学法对中学生多元识读能力 培养的中学美术教学研究奠定基础, 如此在相应程度 上还可以激发师生在美术课堂中互动的热情。经典艺 术历经几千年的改革与传承, 承载着多元的内核精神 能量, 绘画艺术表现形式丰盈多样, 展现出独特的审 美价值, 触发中学生进行创作灵感。

\section{3. 丹培拉教学法对中学生多元识读能力培养 的可行性}

当前中学美术教育教学地位不受重视。中学美术 教学, 由于受应试教育的影响, 大部分师生对美术教 学不够重视。导致学生学习美术不系统, 难以培养审 美的能力和情感。美术教育识读在现在美术课堂中多 表现为图像识读、美术表现、审美判断、创意实践和 文化理解五个基本方向。在全球化的今天这样的学习 目标是远远不足的, 学生美术教育应该与其他的各门 类学科相互联系, 多元化交融结合, 所以丹培拉教学 法对中学生多元识读能力培养具有可行性。

\section{1. 相关国家政策支持}

2020 中共中央办公厅、国务院办公厅印发了《关 于全面加强和改进新时代学校美育工作的意见》 ${ }^{[5]}$ 针 对认识误区和实践问题: 片面、孤立地进行知识技能 教学, 或刻意淡化知识技能教学; 学生艺术实践机会 稀少, 艺术审美体验缺乏提出了批判。指出艺术基础 知识基本技能的教学一定要结合学生艺术审美实践 体验来进行, 并在此基础上形成艺术专项特长。通过 丹培拉绘画材料结合学生自身的艺术基础素养美术 课堂上进行艺术实践, 丹培拉材料操作性强, 容易展 现丰富多变的效果和强大的艺术魅力, 能够在中学美 术课堂教学中提高学生的审美认知。

\section{2. 丹培拉教学法在中学美术课堂的可行性}

丹培拉泛指水和油组成的不透明乳状液体, 常单 用于鸡蛋等乳性胶结合剂组成的绘画。[6]中学美术教 学非常重视培养学生的创造能力, 尤其是在当前新课 程改革的条件下, 中学美术课堂更加重视艺术美学的 学习与创造, 不但让中学生更进一步了解美术, 还让 美术教学更加吸引学生注意力, 开发学生智慧和创造 力。本文将丹培拉绘画语言艺术带进中学美术课堂, 再以丹培拉绘画技法研究美术创作, 让学生的美术作 品更具审美价值。这不但能培养学生的创造力, 还能 提高学生的艺术审美, 有助于提高学生的综合素质能 力。综上所述, 丹培拉绘画对于中学生的美术绘画技 能水平来说是具有一定实践教学可行性。将丹培拉这 一特殊的绘画技法融入美术教育识读中，促使中学生 能在美术课堂中从绘画原始材料出发, 追溯到油画绘 画历史源头, 有助于中学生深入了解绘画材料的特性 特质, 以便于更好地利用绘画材料语言表现形式进行 独到的艺术绘画创作。

丹培拉绘画之所以成为备受关注的艺术绘画语 言, 是因为丹培拉特殊绘画材料的独特性、艺术性、 文化性, 对中学教师的教学研究和师生绘画探索有重 要的学术价值。丹培拉绘画过程中, 油和水还有蛋黄 液以及颜料之间的画面反映多种多样，可以说是各有 不一, 这也就是教学过程中能透过这不同的画面反映 进行对丹培拉材料的理解。丹培拉绘画创作的整个过 程都能提高学生的动手能力及创造能力, 并且能对学 生的艺术素养增色添彩。基于丹培拉绘画材料的种种 优点, 使中国艺术家对此进行大力支持和学习研究, 这更说明中学美术教师乃至于中学生对于这一特殊 绘画材料的学习是有必要的。

\section{4. 丹培拉对多元识读能力的培养}

丹培拉教学法对中学生多元识读能力的培养在 中学美术教学中应用并不仅仅局限于对材料的质感、 
肌理等方面的因素掌握, 而是要让学生掌握具体方法, 并明白知识的学习和技能的完善并不是孤立的, 是要 和生活的实践相互联系。

\section{1. 丹培拉教学法对多元识读的重要性}

在丹培拉教学过程中对于中学美术识读有重要 作用。如何在中学美术识读中应用丹培拉教学, 以下 分两点论述。第一, 要加强中学美术师资问题, 中学 学校应定期在美术教师中开展关于美术专业学术探 讨交流活动, 学习与丹培拉绘画相关的文化知识和丹 培拉材料使用绘画技能, 优化师资艺术素养和审美水 平, 促进中学美术教学水平。此外, 还要增添美术课 时安排, 强调中学生动手使用材料进行绘画创作, 而 并非将美术课当作简单的图像识读鉴赏课。第二, 解 决丹培拉教学设计教材问题, 当前市面上的中学美术 教材中与油画相关的内容相对 较少且并不完善, 再 深入探讨丹培拉这一绘画的更是鲜有。所以中学美术 教师要充分学习与丹培拉绘画相关的素材与艺术资 料来增加美术课堂教学的生动性和活泼性, 将其中的 艺术知识养分传输给中学生, 作为导向可培养中学生 发挥自身潜力和动手能力, 进一步学习丹培拉绘画技 法等传统艺术文化知识。丹培拉教学设计在中学美术 识读的应用, 就在于美术教师通过图像识图先让中学 生了解何为丹培拉, 观看丹培拉教学法的 MISS 录影 可促进教师与学生在美术课堂的教与学的整个过程。

\section{2. 研究方法「TIMSS 录影分析」}

丹培拉教学法对中学生多元识读能力的培养选 用录影分析法, 对中学美术课堂教学录像提供的丰富 而又复杂的信息进行相应的结构化处理, 即知识的外 化, 使之产生能用之于分析教学过程的新知识, 这一 过程称之为录影分析。课堂教学录像分析的基本目标 是构建课堂教学质量的概念, 这与美术课程标准 ${ }^{[7]}$ 直 接有关, 其次是能有效地反映美术课堂教学的真实情 况。因此, 录影分析包括两方面的任务, 既要对教师 教学设计的内容进行分析, 又要对教学过程中实时发 生的事件进行记录分析。TIMSS[8]目的是利用对中学 生的学习成就、课堂参与度、态度的估量, 进行比较 分析。TIMSS 形成如下概念框架图:

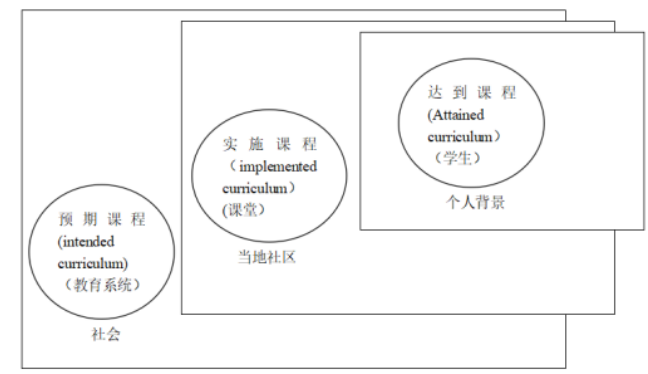

图 1 TIMSS 概念框图
如图显示, 课堂上中学生的学习成果必须在不同 的情境层次中显示: 地方或学校情境中, 在教育情境 中, 预期和事实的课程是中学生教育过程中质量的重 要因素, 它们涵盖了整个教学过程和学习经验, 美术 教师可以运用这些经验来培养中学生的美术学习能 力。在实施过程中, 学生的学习成果不局限于通过完 成美术作业这一学习目标, 还涉及中学生对于美术课 堂态度评价、更高层次的思维能力以及美术技能表现。

\section{3. 「美术基础」课堂教学之示例分析}

美术基础教学 ${ }^{[9]}$ 内容根据分析的需求, 课的内容 分为不同类别, 以艺术理论教学为主, 以材料制作动 手实践为主的, 以录像鉴赏分析学习为主的等。丹培 拉材料创作实践课, 采用丹培拉特殊材料在课堂上给 学生进行动手实操。在课堂教学过程中遇到问题及时 与学生交流互动, 在材料实践中更好的促进师生互动, 激发学生的兴趣。作品完成后, 至少用一节时间在全 班进行展览分享，提高学生的美术审美鉴赏能力，在 作品的要求上尽量注重学生自身的创意及思想。课后 通过 TIMSS 录像分析使学生与教师可在课堂外能继 续学习丹培拉教学法培养多元识读能力。MISS 录像 分析把学生放在本位, 丹培拉教学法理念在美术教学 课堂中的多元识读的培养能更好的改善学生与教师 的教与学, 提高美术学科学习成效和课堂教学质量。 关于 TIMSS 录像分析法与丹培拉教学法之间的联系 如图:

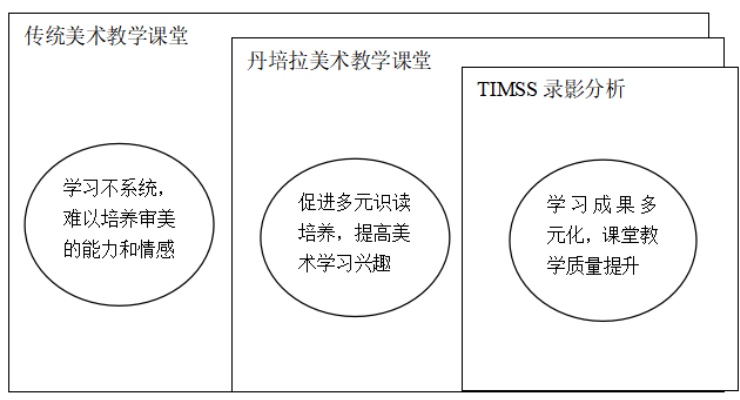

图 2 TIMSS 与美育的联系

如图所示: 在传统美术课堂与丹培拉美术教学课 堂再到 TIMSS 录影分析这三种情境中, 得到的反馈 大有不同, 丹培拉美术教学课堂对中学生多元识读的 培养确有积极作用, 促进有效教学 ${ }^{[1]}$ 。透过 TIMSS 录 像分析美术学科的学习成果更加多元化, 教学质量也 得到了提升。以下是本次研究的技术研究路线图: 


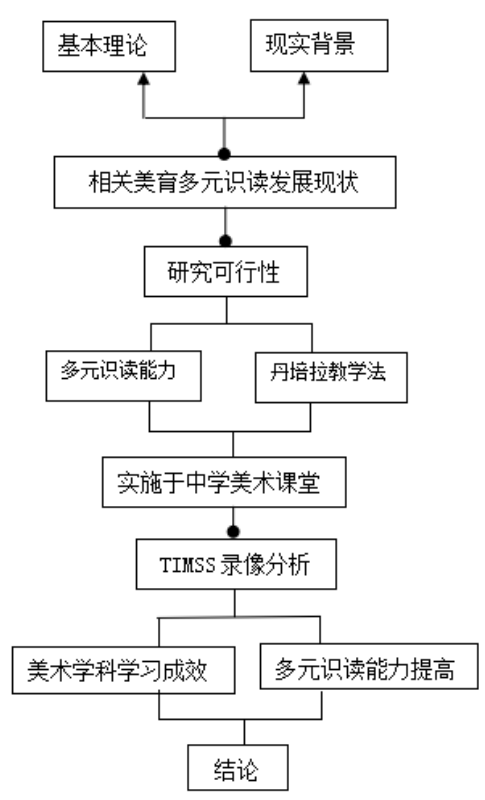

图 3 技术研究路线图

\section{5. 结论}

美术教育应该从传统的教育模式向素质教育模 式发展。在中学美术教育中, 素质教育体现在美术教 育的多个方面, 故丹培拉教学法对中学生多元识读能 力的培养是中学生对美术学习一大重要影响举措。传 统美术学习情境: 美术教师给与范例图让学生观看学 习, 再让学生使用水彩笔蜡笔画纸等简单的美术作画 工具进行作画, 课后上交作业。使用丹培拉教学法进 行美术学习情境: 先让学生通过美术鉴赏了解什么是 丹培拉, 再了解丹培拉的材料, 让学生在材料中了解 学习丹培拉的艺术魅力并且产生艺术创作兴趣。待到 兴趣激发后授予用丹培拉特殊材料的创作方法, 在课 堂上进行创作实践, 在实践中交流学习与研究。前者 与后者的差异在于, 传统美术学习情境枯燥乏味, 没 有注入美术的理论和价值, 后者激起学生兴趣, 对于 艺术美学有更深一层的理解与学习。

中学美术教学还应充分应用时代所带来的互联 网应用优势, 将丹培拉的发展历史及如何创作的相关 资料, 并通过图片、影像等方式呈现出来共享到课堂 上, 以便于学生更直观了解学习, 通过图像识图的教 学方法让学生通过视听感官更直接的产生兴趣, 由此 达到更深层次的升华。综上, 在中学美术教学过程中, 美术教师就需要有意识地导入丹培拉艺术元素, 鼓励 学生就此对于绘画创作进行发散性头脑风暴只有这 样, 学生在学习和应用时, 才能提升自己的创造思维 和创新能力, 提高学生自身的综合素质。概括地说, 丹培拉教学法让中学生从绘画原始材料出发, 回到源 头, 只有深入了解材料的特性特质, 才能更好地利用 材料语言表现形式进行独到的艺术创作, 也才能真正 拥有美术素养。

\section{REFERENCES}

[1] Thwaites, T. Multiliteracies: A new paradigm for arts education [J]. ACEPapers, 2003 (4) : 14-16.

[2] Zhang Hong.Thinking about painting language[J].Art,1994(03):67-69. (Chinese)

[3] Ge Yue. Image Art Language in Modern Visual Communication[D].Sichuan Academy of Fine Arts,2005. (Chinese)

[4] Du Wei.On Aesthetic Quality and Its Cultivation[J].educational Research,2014,35(11):24-31. (Chinese)

[5] General Office of the Central Committee of the Communist Party of China General Office of the State Council Print and distribute"Opinions on Comprehensively Strengthening and Improving School Aesthetic Education in the New Era" $[\mathrm{J}]$.Communiqué of the State Council of the People's Republic of China,2020(30):20-26. (Chinese)

[6] Wang Xinman.On the characteristics and application of materials in Tempera paintings[J].Artist,2020(04):27. (Chinese)

[7] Wang Ding. Research on International Large-scale Mathematics Evaluation[D].Shanghai Normal University,2016 (Chinese)

[8] General high school art curriculum standards[M]. People's education Press , Ministry of Education of the People's Republic of China, 2018 (Chinese)

[9] Wang Guanying.Basic art teaching should focus on cultivating students' aesthetic ability[J].Journal of Jilin Normal University.1996(06) (Chinese)

[10] Choi Yunjong.Effective teaching[M]. East China Normal University Press,2009 (Chinese) 